PROCEEDINGS OF THE

AMERICAN MATHEMATICAL SOCIETY

Volume 141, Number 6, June 2013, Pages 2081-2083

S 0002-9939(2013)11485-1

Article electronically published on January 17, 2013

\title{
A MARCINKIEWICZ MAXIMAL-MULTIPLIER THEOREM
}

\author{
RICHARD OBERLIN
}

(Communicated by Michael T. Lacey)

\begin{abstract}
For $r<2$, we prove the boundedness of a maximal operator
\end{abstract} formed by applying all multipliers $m$ with $\|m\|_{V^{r}} \leq 1$ to a given function.

\section{INTRODUCTION}

Given an exponent $r$ and a function $f$ defined on $\mathbb{R}$, consider the $r$-variation norm

$$
\|f\|_{V^{r}}=\|f\|_{L^{\infty}}+\sup _{N, \xi_{0}<\cdots<\xi_{N}}\left(\sum_{i=1}^{N}\left|f\left(\xi_{i}\right)-f\left(\xi_{i-1}\right)\right|^{r}\right)^{1 / r},
$$

where the supremum is over all strictly increasing finite length sequences of real numbers.

The classical Marcinkiewicz multiplier theorem states that if $r=1$ and a function $m$ is of bounded $r$-variation uniformly on dyadic shells, then $m$ is an $L^{p}$ multiplier for $1<p<\infty$ and

$$
\left\|(m \hat{f})^{\sim}\right\|_{L^{p}} \leq C_{p, r} \sup _{k \in \mathbb{Z}}\left\|1_{D_{k}} m\right\|_{V^{r}}\|f\|_{L^{p}}
$$

where $D_{k}=\left[-2^{k+1},-2^{k}\right) \cup\left(2^{k}, 2^{k+1}\right]$ and, , denote the Fourier-transform and its inverse. Later, Coifman, Rubio de Francia, and Semmes [2] (see also 8]) showed that the requirement of bounded 1-variation can be relaxed to allow for functions of bounded 2-variation, and in fact (1.1) holds whenever $r \geq 2$ and $\left|\frac{1}{p}-\frac{1}{2}\right|<\frac{1}{r}$.

The estimate 2 does not discriminate between multipliers of bounded 2-variation and those of bounded $r$-variation where $r<2$, and so one might ask whether there is anything to be gained by controlling the variation norm of multipliers in the latter range of exponents.

Defining the maximal-multiplier operator

$$
\mathcal{M}_{r}[f](x)=\sup _{m:\|m\|_{V^{r} \leq 1}}\left|(m \hat{f})^{\curlyvee}(x)\right|,
$$

where the supremum is over all functions in the $V^{r}$ unit ball, we have

Theorem 1.1. Suppose $1 \leq r<2$ and $r<p<\infty$. Then

$$
\left\|\mathcal{M}_{r}[f]\right\|_{L^{p}} \leq C_{p, r}\|f\|_{L^{p}}
$$

Received by the editors October 4, 2011.

2010 Mathematics Subject Classification. Primary 42A45; Secondary 42A20.

The author is supported in part by NSF Grant DMS-1068523.

(C) 2013 American Mathematical Society Reverts to public domain 28 years from publication 
The case $r=1$ was observed independently by Lacey [4].

Note that in the definition of $\mathcal{M}_{r}$, each $m$ is required to have finite $r$-variation on all of $\mathbb{R}$ rather than simply on each dyadic shell as in (1.1). This is necessary for boundedness, as can be seen from the counterexamples of Christ, Grafakos, Honzík and Seeger 1 .

Although the maximal operator (1.2) would seem to be fairly strong, we do not yet know of an application for the bound above. We will, however, quickly illustrate a strategy for its use that falls an (important) $\epsilon$ short of success. Let $\Psi$ be (say) a Schwartz function, and for each $\xi, x \in \mathbb{R}$ and $k \in \mathbb{Z}$, consider the $2^{k}$-truncated partial Fourier integral

$$
\mathcal{S}_{k}[f](\xi, x)=p . v \cdot \int f(x-t) e^{2 \pi i \xi t} \Psi\left(2^{-k} t\right) \frac{1}{t} d t .
$$

It was proven by Demeter, Lacey, Tao, and Thiele [3] that for $q=2$ and $1<p<\infty$,

$$
\left\|\sup _{\|g\|_{L^{q}=1}}\right\| \sup _{k}\left|\left(\mathcal{S}_{k}[f](\cdot, x) \hat{g}\right)^{`}\right|\left\|_{L^{q}}\right\|_{L_{x}^{p}} \leq C_{p, q}\|f\|_{L^{p}} .
$$

If we had the bound

$$
\left\|\mathcal{S}_{k}[f](\xi, x)\right\|_{L_{x}^{p}\left(\ell_{k}^{\infty}\left(V_{\xi}^{r}\right)\right)} \leq C_{p, r}\|f\|_{L^{p}}
$$

for some $r<2$, then an application of Theorem 1.1 would give (1.4) for $q>r$ by rather different means than [3]. In fact, one can see by applying the method in Appendix D of [6] that (1.5) holds for $r>2$ and $p>r^{\prime}$. Unfortunately, it does fail for $r \leq 2$.

\section{Proof of Theorem 1.1}

The following lemma was proven in [2]; see also [5].

Lemma 2.1. Let $m$ be a compactly supported function on $\mathbb{R}$ of bounded $r$-variation for some $1 \leq r<\infty$. Then for each integer $j \geq 0$, one can find a collection $\Upsilon_{j}$ of pairwise disjoint subintervals of $\mathbb{R}$ and coefficients $\left\{b_{v}\right\}_{v \in \Upsilon_{j}} \subset \mathbb{R}$ so that $\left|\Upsilon_{j}\right| \leq 2^{j}$, $\left|b_{v}\right| \leq 2^{-j / r}\|m\|_{V_{r}}$, and

$$
m=\sum_{j \geq 0} \sum_{v \in \Upsilon_{j}} b_{v} 1_{v},
$$

where the sum in $j$ converges uniformly.

The lemma above was applied in concert with Rubio de Francia's square function estimate [7] to obtain (1.1). Here, we will argue similarly, exploiting the analogy between the Rubio de Francia square function estimate and the variation-norm Carleson theorem.

It was proven in [7] that for $p \geq 2$,

$$
\sup _{\mathcal{I}}\left\|\left(\sum_{I \in \mathcal{I}}\left|\left(1_{I} \hat{f}\right)^{\curlyvee}\right|^{2}\right)^{1 / 2}\right\|_{L^{p}} \leq C_{p}\|f\|_{L^{p}}
$$

where the supremum above is over all collections of pairwise disjoint subintervals of $\mathbb{R}$. Consider the partial Fourier integral

$$
\mathcal{S}[f](\xi, x)=\left(1_{(\infty, \xi]} \hat{f}\right)^{\curlyvee}(x) .
$$


It was proven in [6] that for $s>2$ and $p>s^{\prime}$,

$$
\|\mathcal{S}[f](\xi, x)\|_{L_{x}^{p}\left(V_{\xi}^{s}\right)} \leq C_{p, s}\|f\|_{L^{p}}
$$

or, equivalently,

$$
\left\|\sup _{\mathcal{I}}\left(\sum_{I \in \mathcal{I}}\left|\left(1_{I} \hat{f}\right)^{\curlyvee}\right|^{s}\right)^{1 / s}\right\|_{L^{p}} \leq C_{p, s}\|f\|_{L^{p}} .
$$

Note that by standardizing limiting arguments, taking the supremum in (1.2) to be over all compactly supported $m$ such that $\|m\|_{V^{r}} \leq 1$ does not change the definition of $\mathcal{M}_{r}$. Applying the decomposition (2.1), we see that for any compactly supported $m$ with $\|m\|_{V^{r}} \leq 1$ we have

$$
\begin{aligned}
\left|(m \hat{f})^{\frown}(x)\right| & \leq \sum_{j \geq 0} \sum_{v \in \Upsilon_{j}}\left|b_{v}\left(1_{v} \hat{f}\right)^{\frown}(x)\right| \\
& \leq \sum_{j \geq 0} \sup _{v \in \Upsilon_{j}}\left|b_{v}\right|\left|\Upsilon_{j}\right|^{\frac{1}{s^{\prime}}}\left(\sum_{v \in \Upsilon_{j}}\left|\left(1_{v} \hat{f}\right)^{\curlyvee}(x)\right|^{s}\right)^{1 / s} \\
& \leq C_{r, s} \sup _{\mathcal{I}}\left(\sum_{I \in \mathcal{I}}\left|\left(1_{I} \hat{f}\right)^{\curlyvee}(x)\right|^{s}\right)^{1 / s}
\end{aligned}
$$

where, for the last inequality, we require $s<r^{\prime}$.

Provided that $r<2$ and $p>r$ we can choose an $s<r^{\prime}$ with $s>2$ and $p>s^{\prime}$, giving (2.2) and hence (1.3).

The argument of Lacey [4] for $r=1$ follows a similar pattern, except with Marcinkiewicz's method in place of [2] and the standard Carleson-Hunt theorem in place of [6].

\section{REFERENCES}

1. Michael Christ, Loukas Grafakos, Petr Honzík, and Andreas Seeger, Maximal functions associated with Fourier multipliers of Mikhlin-Hörmander type, Math. Z. 249 (2005), no. 1, 223-240. MR2106977(2005h:42024)

2. Ronald Coifman, José Luis Rubio de Francia, and Stephen Semmes, Multiplicateurs de Fourier de $L^{p}(\mathbf{R})$ et estimations quadratiques, C. R. Acad. Sci. Paris Sér. I Math. 306 (1988), no. 8, 351-354. MR934617 (89e:42009)

3. Ciprian Demeter, Michael T. Lacey, Terence Tao, and Christoph Thiele, Breaking the duality in the return times theorem, Duke Math. J. 143 (2008), no. 2, 281-355. MR2420509

4. Michael T. Lacey, personal communication.

5. Issues related to Rubio de Francia's Littlewood-Paley inequality, NYJM Monographs, vol. 2, State University of New York, University at Albany, Albany, NY, 2007. MR 2293255 (2007k:42048)

6. Richard Oberlin, Andreas Seeger, Terence Tao, Christoph Thiele, and James Wright, A variation norm Carleson theorem, J. Eur. Math. Soc. 14 (2012), no. 2, 421-464. MR.2881301

7. José L. Rubio de Francia, A Littlewood-Paley inequality for arbitrary intervals, Rev. Mat. Iberoamericana 1 (1985), no. 2, 1-14. MR850681 (87j:42057)

8. Terence Tao and James Wright, Endpoint multiplier theorems of Marcinkiewicz type, Rev. Mat. Iberoamericana 17 (2001), no. 3, 521-558. MR1900894 (2003e:42014)

Department of Mathematics, Louisiana State University, Baton Rouge, Louisiana 70803-4918

E-mail address: oberlin@math.lsu.edu 